\title{
The migration and invasion of human prostate cancer cell lines involves CD151 expression
}

\author{
JIAN ANG ${ }^{1}$, BING-LIANG FANG ${ }^{3}$, LEONIE K. ASHMAN ${ }^{2}$ and ALBERT G. FRAUMAN ${ }^{1}$ \\ ${ }^{1}$ Department of Medicine, Clinical Pharmacology and Therapeutics Unit, Austin Health, University of Melbourne, VIC; \\ ${ }^{2}$ Experimental Oncology Unit, University of Newcastle, NSW, Australia; ${ }^{3}$ Department of Thoracic and \\ Cardiovascular Surgery, MD Anderson Cancer Centre, University of Texas, Houston, TX, USA
}

Received June 4, 2010; Accepted July 16, 2010

DOI: $10.3892 /$ or_00001022

\begin{abstract}
The molecular mechanisms underlying prostate cancer metastasis remain poorly understood. The tetraspanin family member CD151 has been reported as an 'adaptor' between integrins and signal pathways. The role of CD151 in prostate cancer metastasis in vitro was investigated in this study. LNCap cells were transfected with wild-type CD151 cDNA, mutated CD151 cDNA and vector cDNA. The mutant (QRD ${ }^{194-196}$ to INF) CD151 cDNA was created using QuickChange 2 site directed Mutagenesis kit (Stratagene). siRNAs were also used to knock down the CD151 expression in the prostate cancer cell line PC3. Proliferation, migration and invasion properties were measured after gene transfection and gene knock-down. There was no difference in proliferation of untransfected or control transfected LNCap cells vs. CD151 transfected LNCap cells $(\mathrm{P}>0.05)$. There was greater motility of CD151-transfected vs. control cells, when transferring through migration chambers with or without matrigel-coated membranes $(\mathrm{P}<0.01, \mathrm{P}<0.01)$. Fewer numbers of mutant-transfected cells were found on the membranes for both migration and invasion studies $(\mathrm{P}<0.01, \mathrm{P}<0.01)$. CD151 knock-down PC3 cells showed decreased motility $(\mathrm{P}<0.01)$, but no change in proliferation $(\mathrm{P}>0.05)$. Our data show that CD151 does not change the proliferative properties of prostate cancer cells, but does promote migration and invasion, and suggest that CD151 plays a specific role in promoting prostate cancer cell motility.
\end{abstract}

\section{Introduction}

Due to the increasing use of screening with prostate specific antigen (PSA), $60 \%$ of prostate cancer was discovered when

Correspondence to: Professor Albert G. Frauman, Department of Medicine, Clinical Pharmacology and Therapeutics Unit, Level 5, L\&T Building, University of Melbourne, Austin Health, Studley Road, Heidelberg, VIC 3084, Australia

E-mail: albertf@unimelb.edu.au

Key words: CD151, prostate cancer, migration, invasion it was still confined to the gland itself (1). The primary cause of death of prostate cancer is invasion and metastasis, and metastatic prostate cancer is presently incurable (2). Despite prostate cancer being the most common non-cutaneous malignancy in Western countries, the molecular mechanisms underlying prostate cancer metastasis remain poorly understood.

CD151 is a member of the tetraspanin superfamily. Like other tetraspanins, it assembles with other membrane proteins such as integrins to form functional complexes (3). CD151 stands out among all tetraspanins not only because it plays a key role in tetraspanin-tetraspanin and tetraspanin-integrin networks (4), but also because it has been recently reported as the first member of the tetraspanins to be associated with metastasis of human cancers (5).

In a previous study from our laboratory, it was found that CD151 has an increasing expression pattern in prostate cancer progression, and higher levels of CD151 were associated with poorer prognosis; indeed, CD151 had better predictive value for the clinical outcome of prostate cancer patients than did traditional histological grading (Gleason grading) (6). However, functionally important regions and intracellular effects in relation to cancer invasion are unknown for CD151.

In this study, we sought to demonstrate that CD151 imparts enhanced motility of a prostate cancer cell line in vitro by both overexpression and small interfering RNA (siRNA) knock-down models. We also sought to determine where a CD151-integrin binding site is implicated in this regard. Thus, we created normal and mutated (QRD ${ }^{194-196}$ to INF) (7) CD151 transfected LNCap cell lines and CD151 gene knockdown PC 3 cells. Changes in cell proliferation, migration and invasion were evaluated afterwards.

\section{Materials and methods}

Cell line and gene transfection. All cell lines were obtained from American Type Culture Collection (ATCC). PcDNA3.1+/ Zeo vector (Invitrogen) with the full length human CD151 sequence was generated in Professor Ashman's laboratory. LNCap cells were transfected with CD151 cDNA, mutated CD151 cDNA (see below) and vector only using FuGENE6 transfection reagent (Roche). Cells were incubated with $1 \mu \mathrm{g}$ vector CD151 DNA for $48 \mathrm{~h}$ then selected in media containing Zeocin $(1000 \mu \mathrm{g} / \mathrm{ml})$. Medium was changed every 3-5 days until colonies appeared (10-15 days). 


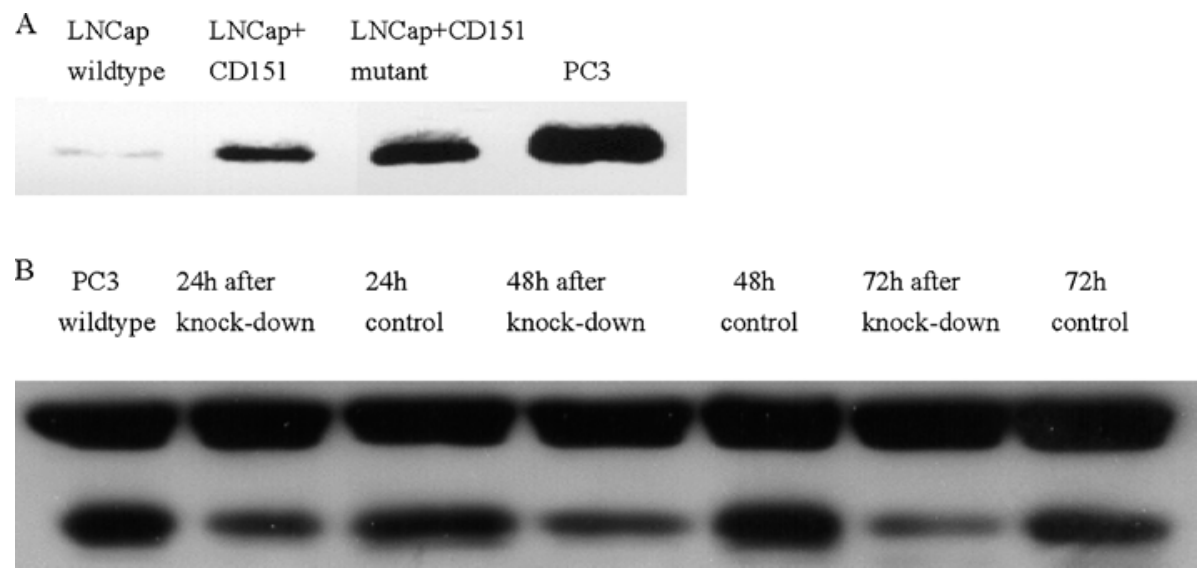

Figure 1. CD151 expression before and after transfection and siRNA knock-down. (A) CD151 protein expression in wild-type LNCap cells, LNCap cells transfected with CD151 cDNA (AC12) and LNCap cells transfected with mutant CD151 cDNA (BC1). (B) The knock-down efficiency of CD151 siRNA in PC3 cells was measured at 24, 48 and $72 \mathrm{~h}$ time-points (upper line, $\beta$-actin; lower line, CD151).

Transfected cells derived from a single clone were chosen and cultured in 98-well plates. CD151 protein expression was measured using Western blotting when enough cells were grown from single clones. Cell lines with the highest CD151 expression were kept for further investigation (Fig. 1).

Generation of CD151 mutant cDNA. CD151-QRD ${ }^{194-196}$ to INF mutant was generated using QuickChange 2 site directed Mutagenesis kit (Stratagene). The CD151 DNA template was denatured at $95^{\circ} \mathrm{C}$. The mutagenic primers containing the desired mutation were annealed at $55^{\circ} \mathrm{C}$ and primers extended using PfuUltra DNA polymerase at $68^{\circ} \mathrm{C}$. The parental DNA was digested with Dpn I enzyme. The pure mutated DNA was transformed into competent cells and harvested. The mutated fragment was confirmed by sequencing.

CD151 gene knock-down using siRNA method. The CD151 siRNA oligos were from Dharmacon (SMARTpool). The oligofectamine transfection reagent (Invitrogen) was used in siRNA transfection. PC3 cells were seeded in 6-well plates (50\% confluent). siRNA reagent was mixed with Opti-MEMI media (Invitrogen) and oligofectamine reagent. The transfection mixture was added to each well afterwards. HamF12K media (500 $\mu \mathrm{l})$ containing $30 \%$ FCS was applied to each well after $4 \mathrm{~h}$. Control siRNA (Dharmacon) and Phosphatebuffered saline (PBS) were used as negative controls. The knock-down efficiency was measured at 24, 48 and $72 \mathrm{~h}$ timepoints with Western blotting. Band intensities were measured using the Histogram function in Adobe Photoshop CS Version 8.0 (Adobe Systems Incorporated, CA, USA). The ratio of $C D 151 / \beta$-actin was generated representing the knockdown efficiency. The $48 \mathrm{~h}$ time-point was chosen for further motility studies (Fig. 1).

Western blot analysis. Proteins extracted from the wild-type and transfected LNCap cells were run on a $12.5 \%$ sodium dodecyl sulfate (SDS) acrylamide gel, transferred to nitrocellulose, and probed by the CD151 monoclonal antibody (8) (Newcastle University) at a 1:500 dilution. The membrane was then incubated with peroxidase linked anti-mouse antibody
(1:4000, Amersham Biosciences). The blots were developed with ECL-film.

Proliferation study. Cell proliferation was measured by trypan blue dye exclusion assay. Cells $\left(1 \times 10^{5}\right)$ were seeded in triplicates in 6-well plates. On days 3 and 6 , cells were trypsinized and counted with the haemocytometer under the microscope. Anchorage-independent growth was carried out using soft agar colony formation assay. LNCap cells were plated in triplicate at 3500 cells per $35-\mathrm{mm}$ dish in DMEM containing $10 \%$ FBS and $0.3 \%$ agar onto a bottom layer of $0.6 \%$ agar. The cells were incubated at $37^{\circ} \mathrm{C}$ for 10 days and the plates were stained with $0.5 \mathrm{ml}$ crystal violet for half an hour. Colonies were then counted, representing anchorageindependent growth of cells. All experiments were repeated in triplicate.

Migration and invasion study. The cell migration and invasion assay was carried out with a cell invasion kit (BD). Cells $\left(5 \times 10^{4}\right)$ were placed over the upper chamber of the inserts in a 24-well tissue culture plate. The invasive cells that migrate through onto the lower surface of membrane (with or without matrigel) were fixed with methanol and stained by crystal violet after $24 \mathrm{~h}$. The stained cells were counted under the microscope at x200 amplification; 5 adjacent fields in each specimen were chosen and counted. In experiments with CD151 knock-down cells, all cells in each specimen were counted after migration and invasion. All experiments were repeated in triplicate. Results were evaluated statistically by one way ANOVA and t-test.

\section{Results}

CD151 expression in prostate cancer cell lines. The expression of CD151 protein was measured by Western blot assay. In two established prostate cancer cell lines, LNCap and PC3, the predicted $28 \mathrm{kDa}$ band (8) was detected. PC3 cells expressed larger amount of CD151 protein compared to LNCap cells (Fig. 1). Therefore, the LNCap cell line was chosen as a suitable model for CD151 overexpression. The 
Table I. Anchorage-independent study with wild-type and CD151 transfected cells.

\begin{tabular}{lcccc}
\hline & $\begin{array}{c}\text { Wild-type LNCap } \\
\text { (Group A) }\end{array}$ & $\begin{array}{c}\text { LNCap + Vector } \\
\text { (Group B) }\end{array}$ & $\begin{array}{c}\text { LNCap + CD151 } \\
\text { (Group C) }\end{array}$ & $\begin{array}{c}\text { LNCap + mutant CD151 } \\
(\text { Group D) }\end{array}$ \\
\hline No. of colonies & $111.7 \pm 10.79$ & $103.0 \pm 7.81$ & $112.0 \pm 3.61$ & $67.3 \pm 8.51$ \\
P-values & $\begin{array}{c}\text { Group AB, AC P }>0.05 \\
\text { Group AD P }<0.01\end{array}$ & $\begin{array}{c}\text { Group BA, BC P }>0.05 \\
\text { Group BD P }<0.01\end{array}$ & $\begin{array}{c}\text { Group CA, CB P }>0.05 \\
\text { Group CD P }<0.01\end{array}$ & Group DA, DB, DC P<0.01 \\
\hline
\end{tabular}

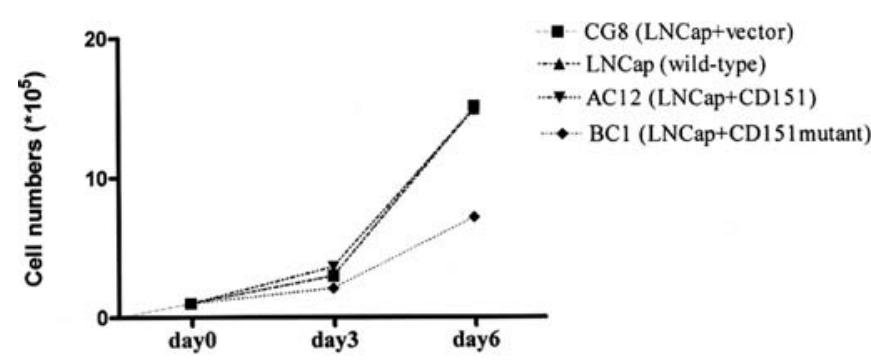

Figure 2. Proliferation study (trypan blue dye exclusion assay). There was no difference between the growing rates of the wild-type LNCap cells and CD151 transfected cells (AC12) $(\mathrm{P}>0.05)$. The CD151-mutant transfected cells $(\mathrm{BC} 1)$ appeared to grow more slowly compared to the other cell lines $(\mathrm{P}<0.01)$.

CD151 transfected cell lines AC12, AE11 and CD151 mutant transfected cell lines BC1, BG7 were used in functional studies (Outcomes with cell lines AE11, BG7 was not shown in this report due to similar statistic results). PC3 cells, expressing high native levels of CD151, were used for CD151 gene knock-down. The CD151 knock-down cells expressed less CD151 protein, reduced by 53, 65 and $68 \%$ at 24,48 and $72 \mathrm{~h}$ time-points (Fig. 1).

CD151 does not accelerate the proliferation of LNCap cells. There was no difference between the growing rates of the wild-type LNCap cells and CD151 transfected cells in trypan blue dye exclusion assay ( $\mathrm{P}>0.05$, Fig. 2). In anchorageindependent growth assay, cells transfected with CD151 gene did not change their character in forming colonies in semisolid agar $(\mathrm{P}>0.05)$. Interestingly, LNCap cells transfected with the CD151-mutant appeared to grow more slowly and formed reduced number of colonies compared to other cell lines $(\mathrm{P}<0.01)$ (Table I).

CD151 gene overexpression promotes the migration and invasion of LNCap cell lines. In migration and invasion study, there were more CD151-transfected cells on both control membrane and matrigel-coated membranes $(\mathrm{P}<0.01, \mathrm{P}<0.01)$ (Figs. 3 and 4), which suggests that CD151 promotes cell migration and invasion. The increasing ratio is even higher for invasion than that of migration $(\mathrm{P}<0.01, \mathrm{P}<0.01)$. The mutant-transfected cells had fewer cells on the membranes in both migration and invasion studies $(\mathrm{P}<0.01, \mathrm{P}<0.01)$ (Figs. 3 and 4).

Knock-down of the CD151 gene inhibits the migration and invasion of prostate cancer cell line PC3. We created a
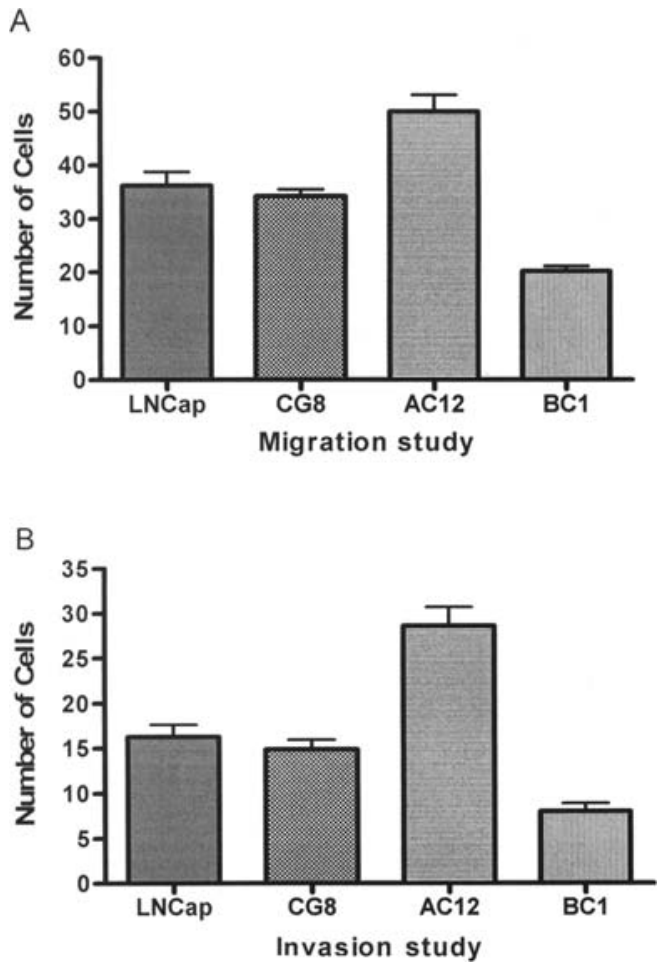

Figure 3. In migration (A) and invasion (B) studies, there were more CD151 transfected cells (AC12) transferred through the matrigel to the other side of membrane compared to wild-type LNCap cells and cells transfected with vector alone (CG8). However, there were fewer mutant-transfected cells (BC1) moved to the other side of the membrane compared to the other cell lines.

CD151 knock-down model in prostate cancer cell line PC3 using CD151 siRNAs. The changes in migration and invasion properties were investigated in these cells. It was found that CD151 knock-down cells had a decreased ability of migration and invasion compared to control siRNA and no siRNA transfected PC3 cells $(\mathrm{P}<0.01, \mathrm{P}<0.01)$ (Fig. 5), however, proliferation was not affected $(\mathrm{P}>0.05, \mathrm{P}>0.05)$ (unpublished data).

\section{Discussion}

High expression of CD151 has been shown to correlate with poor survival rates in prostate cancer (6), colon cancer (9) and lung cancer patients (10). However, its role and mechanisms of action in tumourigenesis and metastasis is still a mystery. 


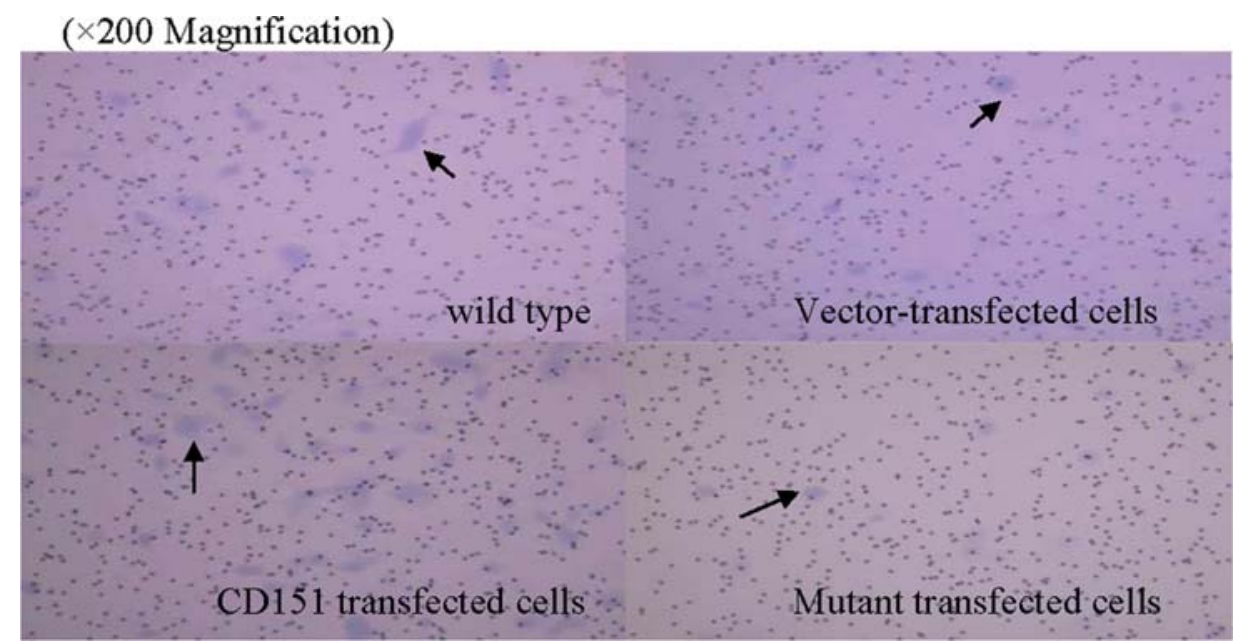

Figure 4. The wild-type, vector transfected, CD151 transfected and mutant transfected LNCap cells transferred to the other side of the membrane under microscope.

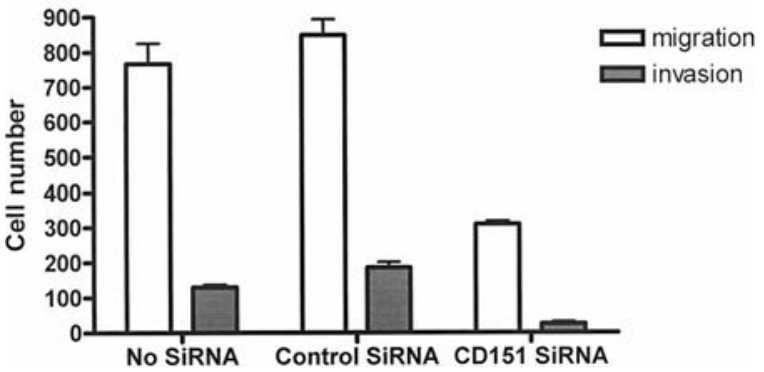

Figure 5. CD151 gene knock-down PC3 cells in migration and invasion studies. It showed a decreased number of CD151 siRNA knock-down cells transferred to the other side of the membrane compared to controls $(\mathrm{P}<0.01)$.

As a key protein in tetraspanin-integrin association, the function of CD151 has been evaluated using overexpressed models in several cancer cell lines $(5,11)$. It has been found that CD151 did have an effect on cancer metastasis in some cell lines $(12,13)$. Testa et al reported that human cervical carcinoma cells transfected with overexpressed CD151 were more migratory than control transfectants expressing low levels of CD151 (5). Kohno et al tested the invasive ability of glioblastoma, colon cancer and fibrosarcoma cell lines into matrigel. They found the cell motility and invasion of CD151-overexpressed cancer cells were markedly enhanced compared to those of the control parental cells, and the numbers of lung metastatic nodules increased significantly in vivo (11). Moreover, the increased migration and invasion properties in transfected cell lines could be reversed by a CD151 monoclonal antibody $(5,11,13)$.

New evidence suggests that tetraspanins may act as linkers between extracellular integrin alpha chain domains and intracellular signalling molecules, such as phosphatidylinositol 4-kinase (PtdIn 4-kinase) and protein kinase C (PKC) (14). The tetraspanin proteins may function as 'adaptors' or 'molecular facilitators' to organize various cell surface proteins into a large web (15). CD151-integrin protein complexes have been identified in many different cell types (15); CD151 plays a key role in the tetraspanin-integrin network because it links other tetraspanins to the integrins (16). The large extra-cellular loop of CD151 and the alpha domain of integrins are thought to be involved in their association $(17,18)$.

Further experiments have been carried out to find which specific region is involved in the association between CD151 and integrins. Yauch and colleagues found that the aa 186217 region within the large extracellular loop of CD151 was critical in CD151-integrin connection (17). In 2002, data from the same laboratory further narrowed the region down to aa 194-196 (7). The mutant without this region lost its highly stoichiometric, stable and specific association with integrin $\alpha_{3} \beta_{1}$ and $\alpha_{6} \beta_{1}(7,15)$.

We chose the prostate cancer cell line LNCap in this study because it expresses lower amounts of CD151 protein compared to other prostate cancer cell lines PC3 and Du145 (data not shown). When we transfected the CD151 cDNA into the LNCap cell line, we also generated an aa 194-196 (QRD-INF) (7) mutant and transfected the mutated CD151 cDNA into LNCap cells as another negative control.

To investigate the role of CD151 in tumor cell proliferation, we measured cell growth using both a cell counting assay and an anchorage-independent growth assay. In the cell counting assay, CD151 transfected cells grow at the same rate as wild-type LNCap cells (doubling time $34 \mathrm{~h}$ ). The CD151 transfected cells formed approximately the same amount of colonies as the wild-type cells after 10 days incubation in semisolid agar, except that CD151 transfected cells formed bigger sized colonies (data not shown).

Migration and invasion are crucial properties of tumor cells, which include detaching themselves from original sites, transferring through the extracellular matrix (ECM) and into the blood circulation. We measured the invasive property of cells with matrigel-coated membranes between upper and lower chambers, which block non-invasive cells from migrating through the membrane. Migration properties were tested by control membranes, which do not contain matrigel and the cells can thus move more freely through the membrane. As shown in Figs. 3 and 4, there were more CD151 transfected cells transferred through the matrigel to the other side of mem- 
brane compared to wild-type LNCap cells and cells transfected with vector alone. The same trend was found in the migration study, although the increased invasive property of CD151 transfected LNCap cells was more obvious.

RNA interference (RNAi) is a post-transcriptional process in eukaryotes targeting specific mRNA for degradation by short-interfering RNAs (siRNAs) (19). The RNAi technique carries out a highly specific knock-down effect in target genes (20), which makes it not only a powerful research tool in numerous gene functional studies, but also a potentially useful therapeutic method in diseases such as cancer and virus infections. Winterwood et al recently found that CD151silenced epithelial carcinoma cells had a markedly reduced motility on laminin-5, and the re-expression of CD151 in those cells reversed the motility defect (21).

CD151 gene knock-down PC3 cells were investigated after siRNA oligos transfection in the present studies. It was found that CD151 knock-down cells had a decreased ability of migration and invasion compared to control siRNA and no siRNA transfected PC3 cells. However, proliferation was not altered after CD151 gene knock-down.

Some unexpected results in this study were the functional changes in CD151 mutant transfected LNCap cells. We used the mutant transfected cells as another negative control and did not expect the CD151 mutant transfection would change the behavior of the cells. To our surprise, the mutant transfected cells grew more slowly than the wild-type and CD151 transfected cells. They formed fewer colonies in soft agar and were not as motile as the wild-type and CD151 transfected cells. As the mutated fragment is the most important region involved in CD151-integrin association (7), it is possible that the overexpression of mutant CD151 gene changes this association and may affect the signaling pathway.

Our laboratory has previously demonstrated that CD151 is clinically valuable in predicting prognosis in prostate cancer patients (6). The present study now provides direct evidence that CD151 promotes migration and invasion of prostate cancer cells, suggesting that it plays a role in prostatic cell motility and is thus possibly involved in the metastatic process of prostate cancer.

\section{Acknowledgements}

This study was supported by Dora Lush Biomedical Postgraduate Research Scholarship from Australian National Health and Medical Research Council (NHMRC).

\section{References}

1. Renald de Wit CNS: Cancers of the genitourinary tract. Taylor \& Francis, London and New York, 2004.

2. Plesnicar S: The course of metastatic disease originating from carcinoma of the prostate. Clin Exp Metastasis 3: 103-110, 1985.

3. Hemler ME: Specific tetraspanin functions. J Cell Biol 155: 1103-1107, 2001.

4. Berditchevski F, Gilbert E, Griffiths MR, Fitter S, Ashman L and Jenner SJ: Analysis of the CD151-alpha3beta1 integrin and CD151-tetraspanin interactions by mutagenesis. J Biol Chem 276: 41165-41174, 2001.
5. Testa JE, Brooks PC, Lin JM and Quigley JP: Eukaryotic expression cloning with an antimetastatic monoclonal antibody identifies a tetraspanin (PETA-3/CD151) as an effector of human tumor cell migration and metastasis. Cancer Res 59: 3812-3820, 1999.

6. Ang J, Lijovic M, Ashman LK, Kan K and Frauman AG: CD151 protein expression predicts the clinical outcome of lowgrade primary prostate cancer better than histologic grading: a new prognostic indicator? Cancer Epidemiol Biomarkers Prev 13: 1717-1721, 2004

7. Kazarov AR, Yang X, Stipp CS, Sehgal B and Hemler ME: An extracellular site on tetraspanin CD151 determines alpha3 and alpha6 integrin-dependent cellular morphology. J Cell Biol 158: 1299-1309, 2002

8. Geary SM, Cambareri AC, Sincock PM, Fitter S and Ashman LK: Differential tissue expression of epitopes of the tetraspanin CD151 recognised by monoclonal antibodies. Tissue Antigens 58: 141-153, 2001

9. Hashida H, Takabayashi A, Tokuhara T, et al: Clinical significance of transmembrane 4 superfamily in colon cancer. Br J Cancer 89: 158-167, 2003

10. Tokuhara T, Hasegawa $\mathrm{H}$, Hattori $\mathrm{N}$, et al: Clinical significance of CD151 gene expression in non-small cell lung cancer. Clin Cancer Res 7: 4109-4114, 2001

11. Kohno M, Hasegawa H, Miyake M, Yamamoto T and Fujita S: CD151 enhances cell motility and metastasis of cancer cells in the presence of focal adhesion kinase. Int J Cancer 97: 336-343, 2002.

12. Hasegawa $H$, Nomura $T$, Kishimoto $K$, Yanagisawa $K$ and Fujita S: SFA-1/PETA-3 (CD151), a member of the transmembrane 4 superfamily, associates preferentially with alpha 5 beta 1 integrin and regulates adhesion of human $\mathrm{T}$ cell leukemia virus type 1-infected T cells to fibronectin. J Immunol 161: 3087-3095, 1998.

13. Yanez-Mo M, Alfranca A, Cabanas C, et al: Regulation of endothelial cell motility by complexes of tetraspan molecules CD81/TAPA-1 and CD151/PETA-3 with alpha3 beta1 integrin localized at endothelial lateral junctions. J Cell Biol 141: 791-804, 1998.

14. Hemler ME: Integrin associated proteins. Curr Opin Cell Biol 10: $578-585,1998$.

15. Yauch RL, Berditchevski F, Harler MB, Reichner J and Hemler ME: Highly stoichiometric, stable and specific association of integrin alpha3beta1 with CD151 provides a major link to phosphatidylinositol 4-kinase, and may regulate cell migration. Mol Biol Cell 9: 2751-2765, 1998.

16. Berditchevski F, Odintsova E, Sawada S and Gilbert E: Expression of the palmitoylation-deficient CD151 weakens the association of alpha 3beta 1 integrin with the tetraspanin-enriched microdomains and affects integrin-dependent signaling. J Biol Chem 277: 36991-37000, 2002.

17. Yauch RL, Kazarov AR, Desai B, Lee RT and Hemler ME: Direct extracellular contact between integrin alpha(3)beta(1) and TM4SF protein CD151. J Biol Chem 275: 9230-9238, 2000.

18. Sterk LM, Geuijen CA, Oomen LC, Calafat J, Janssen H and Sonnenberg A: The tetraspan molecule CD151, a novel constituent of hemidesmosomes, associates with the integrin alpha6beta4 and may regulate the spatial organization of hemidesmosomes. J Cell Biol 149: 969-982, 2000.

19. Hammond SM, Caudy AA and Hannon GJ: Post-transcriptional gene silencing by double-stranded RNA. Nat Rev Genet 2: 110-119, 2001.

20. Brummelkamp TR, Bernards R and Agami R: Stable suppression of tumorigenicity by virus-mediated RNA interference. Cancer Cell 2: 243-247, 2002.

21. Winterwood NE, Varzavand A, Meland MN, Ashman LK and Stipp CS: A critical role for tetraspanin CD151 in alpha3beta1 and alpha6beta4 integrin-dependent tumor cell functions on laminin-5. Mol Biol Cell 17: 2707-2721, 2006. 